\title{
Starting the new review series: Pregnancy-Induced Hypertension
}

Hypertension Research (2017) 40, 3-4; doi:10.1038/hr.2016.105

$\mathrm{O}$ one hand the global population is constantly expanding, while on the other hand some developed countries are facing the demographic problem of decreasing population. The latter problem is usually characterized by falling birthrate and aging of the population. In such a societal situation, the importance of maternal and child health is naturally increasing in order to preserve the working population and promote social activity and productivity. This problem definitely requires perinatal care to prevent maternal and infant loss, as well as concerns issues such as infertility treatment and facility of nursery service. Particularly because the ages of parturient women are higher than before in the developed countries, pregnancies and deliveries complicated by aging and lifestyle-related diseases such as hypertension and diabetes are found to be increasing. As a result, medical and general concern towards improvement of maternal and neonatal outcomes is growing. In these circumstances, management of blood pressure abnormalities during the perinatal period is certainly an important subject to be discussed.

Articles on hemodynamic abnormalities, including hypertension during pregnancy, published in our journal, Hypertension Research, have been increasingly drawing the interest of readers and researchers (Figure 1). Considering these social and academic backgrounds, the editorial office has planned to start publishing a series of review articles on hypertension during pregnancy. We expect this new review series entitled 'Pregnancy-Induced Hypertension (PIH)' will

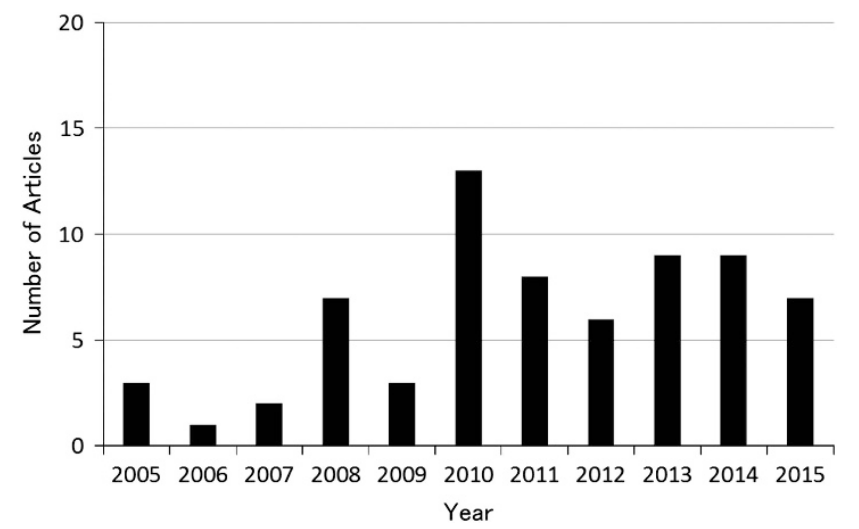

Figure 1 Annual changes in the number of original and review articles published in Hypertension Research regarding pregnancy-induced hypertension. deepen the insight into this topic and provide valuable information for the improvement of blood pressure management and consequent prognosis of mothers and neonates in the perinatal period and thereafter.

Formerly, terms such as 'toxemia of pregnancy' and 'eclampsia' were used for pregnancy complicated by hypertension and related disorders. However, because toxemia is not thought to aptly reflect the pathophysiological condition, and eclampsia corresponds to the extreme stage accompanying convulsion, which rarely occurs nowadays, usage of these words has decreased, although preeclampsia is still commonly used to refer to the gestation hypertension accompanying proteinuria. Instead, the term PIH has come to be generally used and is defined as blood pressure $\geqslant 140 / 90 \mathrm{~mm} \mathrm{Hg}$ after week 20 of pregnancy and returning to normotension within 12 weeks after delivery. However, hypertension during the perinatal period is not necessarily induced by solely pregnancy itself. Pre-existing hypertension and/or renal diseases before pregnancy can be superimposed on pregnancy, and also exert detrimental influences on the prognosis of mothers and newborns. In the Japanese Society of Hypertension Guidelines for the Management of Hypertension (JSH 2014), ${ }^{1}$ the relevant section is headed as 'pregnancy-associated hypertension'; however, PIH is much more frequently used. On the other hand, the International Society for the Study of Hypertension in Pregnancy (ISSHP) has described 'hypertensive disorders of pregnancy' in the latest version of their statement. ${ }^{2}$ The new review series starting in this issue is entitled PIH; however, the term may be possibly varied by the accumulation of experience, researches and evidence in the future. In any case, it should be kept in mind that the disorders to be concurred remain unaltered whatever the term used.

In this series of reviews on $\mathrm{PIH}$, epidemiological information regarding to the current status, such as incidence and prognosis, will be first overviewed. Then, the etiology of PIH is to be thoroughly analyzed in order to delineate the risk factors that are essentially needed for the preventive approach. The pathophysiology of PIH is also required to be figured out for prescribing the effective intervention. Besides, how the blood pressure should be controlled is a matter of great concern to the physicians practicing in the real world. However, available clinical evidence is limited, especially regarding the effects of antihypertensive drugs, because it is ethically difficult to conduct interventional studies in pregnant women. Therefore, the opinions and recommendations of experts in this field, 
based on their experience and consideration, are highly needed, which will also be included in the constitution of this review series.

Toshihiko Ishimitsu

Editor-in-Chief,

Department of Cardiology and Nephrology, Dokkyo Medical

University, Mibu, Japan

E-mail: isimitu@dokkyomed.ac.jp
1 Japanese Society of Hypertension Committee for Guidelines for the Management of Hypertension. The Japanese Society of Hypertension Guidelines for the Management of Hypertension. Hypertens Res 2014; 37: 253-390.

2 Tranquilli AL, Dekker G, Magee L, Roberts J, Sibai BM, Steyn W, Zeeman GG, Brown MA. The classification, diagnosis and management of the hypertensive disorders of pregnancy: a revised statement from the ISSHP. Pregnancy Hypertens 2014; 4: 97-104. 